\title{
Development of Good Modelling Practice for Physiologically Based Pharmacokinetic Models for Use in Risk Assessment: The First Steps
}

George Loizou, ${ }^{1}$ Martin Spendiff, ${ }^{1}$ Hugh Barton, ${ }^{2}$ Jos Bessems, ${ }^{3}$ Frédéric Y. Bois, ${ }^{4}$ Michel Bouvier d'Yvoire, ${ }^{5}$ Harrie Buist, ${ }^{6}$ Harvey Clewell III, ${ }^{7}$ Bette Meek, ${ }^{8}$ Ursula Gundert-Remy, ${ }^{9}$ Walter Schmitt, ${ }^{10}$

${ }^{1}$ Health and Safety Laboratory, Harpur Hill, Buxton, SK17 9JN, UK

${ }^{2}$ National Centre for Computational Toxicology, Office of Research and Development, U.S. Environmental Protection Agency, Research Triangle Park, NC 27711, USA

${ }^{3}$ Centre for Substances and Integrated Risk Assessment (SIR) National Institute for Public Health and the Environment (RIVM), PO Box 1, Bilthoven, 3720 BA, The Netherlands

${ }^{4}$ INERIS, Parc Technologique Alata BP2, 5 rue Taffanel, Verneuil-en-Halatte, 60550, France

${ }^{5}$ ECVAM, EC-Joint Research Centre, via E. Fermi 1, I-21020 Ispra (VA), Italy

6 TNO, P.O.Box 360, Zeist, 3700 AJ, The Netherlands

7 The Hamner Institutes for Health Sciences, P.O Box 12137, 6 Davis Drive, Research Triangle Park, NC, USA

${ }^{8}$ Health Canada, Existing Substances Division, 269 Laurier Avenue West, Ottowa, Ontario, K1A 0K9

${ }^{9}$ BfR, Federal Institute for Risk Assessment, Thielallee 88-92, Berlin, D-14195, Germany

${ }^{10}$ Bayer Technology Services GmbH, Building 6550, Monheim, 40765, Germany

Address correspondence to:

George Loizou

HSL

Computational Toxicology Team

Mathematical Sciences Unit

Harpur Hill

Buxton SK17 9JN

UK

George.loizou@hsl.gov.uk 


\section{Abstract}

The need to develop internationally recognized good modelling practices has resulted from the increasing use of tissue dosimetry estimated using pharmacokinetic models in chemical risk assessments. These practices would facilitate sharing of models and model evaluations and consistent applications in risk assessments. Clear descriptions of good practices for 1) model development (i.e., research and analysis activities), 2) model characterization (i.e., methods to describe how consistent the model is with biology and strengths and limitations of available model and data, such as sensitivity analyses), 3) model documentation, and 4) model evaluation (i.e., independent review), will assist risk assessors, who need to decide whether and how to use the models, but also model developers who need to know what is expected for various purposes (e.g., research versus application in risk assessment). The current status of the application of physiologically based pharmacokinetic (PBPK) models in risk assessments in Europe, Canada, and the United States were reviewed at the International Workshop on the Development of Good Modelling Practice (GMP) for PBPK Models in Greece on April 27 - 29, 2007. The meeting participants identified follow-up steps towards the creation of descriptions of good modelling practices and research to improve the scientific basis of the models.

Key Words: Good modelling practice; PBPK; risk Assessment. 


\section{Introduction}

The increasing use of tissue dosimetry estimated using pharmacokinetic models in chemical risk assessments in multiple countries across the globe is producing greater awareness of the need to develop internationally recognized good modelling practices. These practices would facilitate sharing of models and model evaluations and consistent applications in risk assessments. Clear descriptions of good practices for,

1. model development (i.e., research and analysis activities),

2. model characterization (i.e., methods to describe how consistent the model is with biology and the strengths and limitations of available model and data, e.g., sensitivity analyses),

3. model documentation, and

4. model evaluation (i.e., independent review), would assist not only risk assessors who need to decide whether and how to use the models, but also model developers who need to know what is expected for various purposes (e.g., research versus application in risk assessment) (Cobelli, C. et al. 1984; Portier, C. J. and C. M. Lyles 1996; Rescigno, A. and J. S. Beck 1987).

For risk assessors, good modelling practice would describe a path forward when seeking to evaluate the potential for a pharmacokinetic model, particularly a physiologically based pharmacokinetic (PBPK) model, to contribute to a risk assessment. Good modelling practices would only be one element, however, in a broader context that describes risk assessment practices ranging from limited information analyses (often referred to as default or screening analyses) to biological modelling of the toxicokinetics and toxicodynamic processes in animals and humans. The shift from 'default' through 'data-informed' approaches to risk assessment represents a 'continuum' of methods. The transition begins with default approaches which involve empirical observations made from broad databases of information that were not group, species or chemical specific and where pharmacokinetics and dynamics were not explicitly addressed. The next phase is 'categorical' and 'species-specific' approaches where substances and species are placed into categories based on their characteristics. The categorical approach is followed by the IPCS scheme which uses compound-related and/or chemical specific 
adjustment factors (CSAF) (IPCS 2005), that included chemical-specific pharmacokinetic or pharmacodynamic data. When appropriate, fully data-derived, chemical specific, biologically based dose response risk assessment methods can be employed for chemicals of high concern or with high economic impacts.

Descriptions of known or hypothesized modes of action (i.e., the toxicodynamic process) leading to the toxicity under consideration play key roles throughout much of the continuum of risk assessment methods. A framework for organizing and evaluating evidence supporting modes of action has been described, which is applicable to all toxicity endpoints (Seed, J. et al. 2005; Sonich-Mullin, C. et al. 2001; US EPA 2005). Additional steps to evaluate whether a mode of action would occur in humans based upon what was known from other animal species have also been presented (Boobis, A. R. et al. 2006; Boobis, A. R. et al. 2007; Seed, J. et al. 2005). Application of toxicokinetic and toxicodynamic information in estimating values of factors accounting for interspecies extrapolation and human interindividual variability has been described (Gundert-Remy, U. and C. Sonich-Mullin 2002; IPCS 2005). These factors may be addressed using appropriate data without a pharmacokinetic model or they may be replaced by application of PBPK models.

For modellers, GMPs are important because they describe the kinds of model characterization and documentation that will be considered in a model evaluation process when a model is considered for application in risk assessment. The initial creation of models, along with needed laboratory experimentation, can be a creative and unpredictable process that will be minimally altered by GMPs. However, even at this very early stage, awareness of GMPs can be valuable, including recommendations when publishing models in the peer reviewed literature (Andersen, M. E. et al. 1995). For example, modellers often try several alternative structures as they attempt to reconcile the available data and the description of the biology in the model. These alternatives would not be documented to the same degree as a model proposed for use in risk assessment, but documenting these alternatives were considered important in supporting the model structure eventually selected (Barton, H. A. et al. 2007). 
The International Workshop on the Development of GMP for PBPK models $^{1}$ was convened with two overarching themes:

1. The selection and evaluation of an appropriate deterministic ${ }^{2}$ model structure.

2. Making PBPK techniques accessible to regulators and risk assessors.

This was the first forum dedicated to review, discuss and promote best practice in deterministic PBPK model building and parameterization, including transparency in documentation with clear audit trails for model components, and to facilitate dialogue and understanding between PBPK practitioners and risk assessors and regulators. By bringing together PBPK modellers, mathematicians, statisticians, risk assessors, regulators and laboratory scientists, the sponsors ${ }^{3}$ of this workshop seek international implementation of PBPK modelling in risk assessment, which development of GMP for PBPK should facilitate. This paper presents the results and conclusions of the GMP workshop.

\section{Current Practice - Where do we stand?}

To move forward effectively in developing GMPs, it is useful to understand current practices in PBPK modelling and its application in risk assessment. These applications are more widespread than has been commonly recognized. The range of uses of PBPK models also need to be reflected in appropriate practice reflecting the stage of development or application for the particular model. Efforts to develop GMP for other

\footnotetext{
${ }^{1}$ April 26 - April 28, 2007, at the Mediterranean Agronomic Institute of Chania, Crete, Greece. Presentations, and discussion papers are at http://www.hsl.gov.uk/news/news_pbpk.htm. Additional information is available at www.pbpk.org.

2 A "deterministic" model is the mathematical representation of the biological/chemical system (e.g., PBPK model and metabolic scheme) as opposed to a "non-deterministic" model which is the mathematical/statistical representation of the uncertainty, variability, and covariance of the data and parameters of the deterministic model (e.g., statistical model for measurement errors and population variability).

${ }^{3}$ Sponsors of the International Workshop on the Development of Good Modelling Practice for PBPK models: The UK Health and Safety Executive (HSE), the UK Health and Safety Laboratory (HSL), Health Canada, The European Chemical Industry Council (CEFIC) and The European Centre for the Validation of Alternative Methods (ECVAM).
} 
models applied in environmental regulation can also be informative in terms of their form, content, and processes.

\section{Value of PBPK models in risk assessments}

The need for change of the current risk assessment paradigm is due to an increasing demand from risk assessors and regulators for a higher precision of risk estimates, a greater understanding of uncertainty and variability (Allen, B. C. et al. 1996; Barton, H. A. et al. 1996; Clewell, H. J. et al. 1999; Clewell, H. J. et al. 2002; Cox, L. A., Jr. 1996; Delic, J. I. et al. 2000), a more justifiable means of extrapolating across species, routes, doses and time (Clewell III, H. J. and M. E. Andersen 1987), a more effective means of interpreting biological monitoring data (Georgopoulos, P. G. et al. 1994; Hays, S. M. et al. 2007) and a reduction in reliance on animal testing (Barratt, M. D. et al. 1995; Blaauboer, B. J. et al. 1999; Blaauboer, B. J. et al. 1996; DeJongh, J. et al. 1999). Incorporating PBPK modelling into the risk assessment process can advance all of these objectives. Further, the shift away from standard setting toward a more cost-benefit analysis approach to risk assessment should also see an increase in the utility of biologically based approaches in the support risk management decisions. These topics were addressed in presentations by Dr. George Loizou of the Health and Safety Laboratory, UK in his introduction to the meeting and Dr. Bette Meek of Health Canada in a talk on "Physiologically based pharmacokinetic models (PBPK): the needs of risk assessors". They are also the subject of many paper in the scientific literature as well as reviews (US EPA 2006).

Dr. Meek elaborated on how hazard and dose-response assessment are being driven by mode of action and data informed approaches to characterise dose-response. These evolving developments in risk assessment form the basis for the IPCS harmonization frameworks that, consequently, should also facilitate the incorporation of PBPK modelling into this process. The IPCS harmonization initiative for the risk assessment of chemicals seeks to improve methods through the pursuit of common principles and approaches by drawing on global expertise. It does not seek to standardize the process but to increase understanding and acceptance by identifying potential areas of 
convergence and work towards the development of analytical frameworks, guidance and associated training materials and the sharing of assessments to avoid duplication (Boobis, A. R. et al. 2006; Boobis, A. R. et al. 2007; IPCS 2005; Meek, B. et al. 2003; Meek, M. E. et al. 2003; Meek, M. E. and A. Renwick 2006; Meek, M. E. et al. 2001; Meek, M. E. et al. 2002; Sonich-Mullin, C. et al. 2001).

\section{Current status of implementation of PBPK models in risk assessments}

Dr. Ursula Gundert-Remy described use of PBPK models in the European Union (EU) Existing Substance Regulation (ESR) programme. Under this programme risk assessments are prepared by Rapporteur Member States (RMS) and reported as draft Risk Assessment Reports (RARs). The European Technical Committee then discusses draft RARs on New and Existing Substances (TC NES). Since the introduction of the ESR programme (1996 - 2007), 140 substances have been assessed. Dr. Gundert-Remy briefly reviewed 80 of these chemicals to determine how many risk assessments included the application of PBPK modelling in the 'toxicokinetics' section of the RAR and whether the results of the PBPK analysis influenced the outcome. PBPK modelling was noted in the pharmacokinetic assessment of 8 chemicals (benzene, cyclohexane, acrylic acid, methylmethacrylate, vinyl acetate, 2-butoxyethanol, propylene methyl glycol and styrene). A PBPK model was used and influenced the outcome of the risk assessment in the case of four substances, whereas for another four substances a PBPK model was used but did not influence the outcome. Therefore, PBPK analysis influenced the risk assessment of $5 \%$ of chemicals. The PBPK model for benzene was not used because the mode of action in rodent and human was considered different. The dose metric for acrylic acid was predicted to be 3 times lower in people than in rat, but was still not used. There was no explanation as to why the modelling results for cyclohexane and methylmethacrylate were not used. In the case of vinyl acetate, 2-butoxyethanol, and propylene methyl glycol (1-methoxypropan-2-ol) the interspecies uncertainty factor was reduced based upon analyses using the PBPK models. As result of the quantification of the interspecies differences in glutathione depletion in lung cells, the classification of styrene as a category 2 carcinogen was changed to category 3. 
Dr. Gundert-Remy was unable to discern if the application and acceptance of PBPK models in RARs was dependent upon access to PBPK expertise within any given RMS. The lack of a standardized procedure for the evaluation of a PBPK models and their output was the main concern that prevented application in other chemical risk assessments. Dr. Gundert-Remy was not aware of the approach to evaluation of the PBPK models used in these risk assessments. However, these examples of the use of PBPK modelling by RMS and acceptance by other Member State delegates indicate a shift in risk assessment practices.

In the UK PBPK modelling has been used by the Health and Safety Executive (HSE) in a number of ways in support of its regulatory activities. A PBPK model for formaldehyde was formulated and used to support the position that formaldehyde should not be regarded as an established cause of leukemia in humans and should be considered unlikely to do so (Franks, S. J. 2005). The validity of a biomarker of exposure for 2butoxyethanol was studied using a PBPK model (Franks, S. J. et al. 2006) and the robustness of past regulatory decisions were examined using PBPK models and Monte Carlo sampling (Delic, J. I. et al. 2000).

In France, The French Agency for Environmental and Occupational Health Safety and INERIS are developing the use of PBPK modeling for setting reference values for reproductive toxicants (INERIS, 2007) [full reference: INERIS, 2007, Reprotoxicity of Ethylene Glycol Ethyl Ether (EGEE) in Humans - Development of a Dose-Response Relationship, Report DRC 07-83452-0079A]

PBPK modelling has been used in Canada in the development of chemical specific adjustment factors for several chemicals including chloroform, 2-butoxyethanol, ... (BETTE MEEK WRITE?)

In the United States, PBPK models have been used in safety or risk assessments by several government Agencies including the Food and Drug Administration (FDA), the 
Consumer Products Safety Commission (CPSC), the Occupational Safety and Health Administration (OSHA), and the U.S. Environmental Protection Agency (EPA). As described in the presentation titled "Reducing uncertainty in risk assessment using PBPK Modelling: An example with methylene chloride" by Dr. Melvin E. Andersen of CIIT at The Hamner Institutes for Health Research, modelling for methylene chloride has involved an iterative hypothesis testing process for the pharmacokinetics and glutathione transferase-mediated mode of action leading to cancers in rodents. The mathematical model gave a quantitative form to the researcher's conception of the biological system, permitting the development of a testable, quantitative hypothesis, the design of informative experiments and the ability to recognize inconsistencies between theory (model) and data. The explicit description of model parameters also led to the ability to study and quantify uncertainty. The PBPK model for methylene chloride has been widely applied in risk assessments by CPSC (Babich, M. A. 1998), OSHA for establishing the permissible exposure level including use of Bayesian statistical parameter estimation and characterization of uncertainty and variability (OSHA 1997), and EPA in the IRIS assessment for inhalation cancer risk (Dewoskin, R. S. 2007; US EPA 1987). FDA raised concerns about the potential for teratogenicity of dermally applied all-trans retinoic acid that were addressed by PBPK modelling and evaluation of several potential dose metrics for the active morphogen (Clewell, H. J., 3rd et al. 1997; Rowland, M. et al. 2004). The EPA has used PBPK models for several chemicals in addition to methylene chloride in IRIS assessment (Dewoskin, R. S. et al. 2006). These include the cancer assessment for vinyl chloride and noncancer assessments for vinyl chloride, ethylene glycol monobutyl ether, and xylene. PBPK models have been considered for, but not applied, in the IRIS assessments for acetone, chloroform, and methyl ethyl ketone. IRIS assessments are ongoing for a number of other chemicals that propose to use PBPK models, such as that for trichloroethylene (Chiu, W. A. et al. 2006).

\section{What can we learn from other similar experiences?}

While use of quantitative modelling for toxicology, particularly for biologically based dose-response analyses, has been limited, modelling has been used extensively for 
environmental fate and transport in European, Canadian, and US regulatory contexts. In the US (Canada?, Europe?), risk assessments for specific contaminated sites or permitting of industrial facilities also rely heavily on often complex models for exposure pathways including food chains (US EPA 1989). Interest in reading across chemicals to make predictions has also created efforts to describe some GMPs for quantitative structure activity relationship (QSAR) models. These experiences provide perspectives potentially useful for the development of GMPs for PBPK modelling.

\section{Environmental Modelling- Achieving Acceptance in the Regulatory World}

Dr. Gerhard Goerlitz of Bayer Crop Science who had participated in the process in Europe described the development of good practice in environmental fate modelling. Environmental fate modelling was adopted as a regulatory tool in the European Union (EU) as a result of two major issues. The first was EU legislation in the late 1980s which, set a maximum pesticide residue concentration of $0.1 \mu \mathrm{g} \mathrm{L}^{-1}$ in both drinking and ground water and the second was the very long time, typically 3 to 4 years, required to conduct lysimeter ${ }^{4}$ studies. The latter problem meant that decisions on the identification of critical products and their uses as required by agriculture, while avoiding contamination of groundwater resources, could not be made rapidly. Environmental fate modelling was recognized as a promising approach to address these issues, but questions were raised concerning whether model predictions were sufficiently reliable and how the integrity of model calculations could be ensured. Clear divisions in attitudes emerged following initial discussions among environmental fate modellers, regulators, and registrants. Researchers used the models for the investigation of processes and systems, requiring flexibility and adaptability while maintaining full control of processes and algorithms in the models. Regulators and registrants wanted to predict exceedence or adherence to a regulatory limit, requiring scientific and legal certainty and preferred the use of models for which the code was not subject to alteration, provided complete documentation with clear audit trails for calculations and prevented accidental or intentional misuse. Further

\footnotetext{
${ }^{4}$ The measurement of the water percolating through soils and the determination of the materials dissolved in the water.
} 
conflicts arose because version control and documentation of research models was frequently rudimentary, if it existed, no guidance on the selection of appropriate input parameters was available, and it was rarely properly established whether a model design was really suitable for regulatory purposes. These problems were a reflection of the fact that the typical research model was intended for use by a specialist with specific and intensive training, which at the time was almost totally lacking in regulatory agencies and in companies trying to assess the environmental behavior of plant protection products. The natural consequence was general confusion with different modellers arriving at completely different results while supposedly using the same models for the same problem.

The first attempt to address this problem was a technical quick fix in the form of software packages comprising models and preconfigured scenarios with a user-friendly graphical interface. As a consequence, non-expert users still produced poor results increasing confusion further. There were two main reasons for this, (i) model processes, algorithms and standard parameters did not appropriately reflect substance properties and (ii) substance data from standard environmental fate studies were conceptually different from the model implementations. This led to a proposal to apply good laboratory practice (GLP) for modelling because GLP was supposed to ensure that all data could be traced and reviewed and accidental input of incorrect data, as well as forgery, could be detected. Also, GLP had just been successfully been transferred from toxicology into the metabolism, environmental fate and residue analysis laboratories. On the other hand, measurements are never perfectly reproducible (especially not for living systems) whereas simulations are and GLP is difficult to apply to electronic data systems and calculations. A solution was found in a short document entitled, "Rules for the correct performance and evaluation of model calculations for simulation of the environmental behavior of pesticides". Later to be known as the 'Codex' this document was produced as a result of cooperation between two German regulatory agencies, a research institute and 
the German Agrochemical Industry ${ }^{5}$. The Codex outlined general principles of GMP, not a detailed "cooking recipe" type of guidance. It focused on leaching models but was generally applicable to other simulation models. It covered the following topics:

- Selection of models

- Documentation of models

- Validation

- Support

- Official recognition and version control

- Selection and treatment of input data

- Consistency of input data and models

- Documentation of simulations

- Reporting

- Interpretation

The "Codex" created a basis for the regulatory acceptance of simulation models on the national scale in Germany, as well as forming a platform to address the requirements of the European directive 91/414 ${ }^{6}$. After, several informal meetings between modellers,

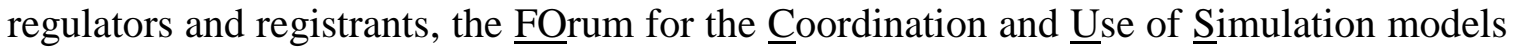
(FOCUS) was created. The steering committee of FOCUS met under the auspices of the EU Directorate General for Health and Consumer Affairs (DG SANCO) for the first time in 1993 and approved 2 research area themes on groundwater and surface water models. FOCUS decisions are based on consensus. It has equal representation of regulators, researchers and industry. The remit is to offer technical support to the EU registration process (91/414). It has no administrative infrastructure, but DG SANCO provides funds for attendance at meetings for regulatory experts and researchers. The FOCUS committee meets approximately 4 times per year and has 2 permanent institutions:

\footnotetext{
${ }^{5}$ Federal Biological Research Centre for Agriculture and Forestry (BBA), the Federal Environmental Agency (UBA), the Fraunhofer Institute for Environmental Chemistry and Ecotoxicology (FhG IUCT) and the German Agrochemical Industry (IVA).

${ }^{6}$ http://europa.eu.int/eur-lex/en/consleg/pdf/1991/en_1991L0414_do_001.pdf
} 
- The FOCUS website ${ }^{7}$ provides all the reports of past FOCUS projects, as well as the actual versions of models which are recommended in the FOCUS reports as well as essential scenario data.

- The Version Control Group is a technical group. Members are the model developers/supporters. Its responsibilities are the approval of new model versions, and the control of the website content. Generally, no formal meetings of the VC are held.

Today the FOCUS reports have achieved a prominent position in the exposure assessment for the registration of plant protection products at the EU level. This is best reflected by the fact that in the present draft of the revision of the EU directive 91/414 on the authorization of plant protection products there are many instances where FOCUS reports are directly referred to as guidance on important decision points. Apart from that many member states use FOCUS outputs and adapted it as guidance in their exposure and risk assessments.

\section{QSAR dossiers}

Recent activities within the EU with regard to the development of templates for the various stages in the application of quantitative structure activity relationships (QSARs) potentially provide a blueprint for good PBPK modelling practice ${ }^{8}$. Templates for QSAR development, prediction and reporting have been proposed. Each template is relevant to different stages of the risk assessment process. In the development template, information on the training domain, internal validation, cross validation and external validation is stored. In the prediction template the substance-specific prediction is stored. Finally, the reporting template should clearly state how the prediction should be used and applied.

\footnotetext{
${ }_{8}^{7}$ http://viso.ei.jrc.it/focus/

http://ecb.jrc.cec.eu.int/documents/REACH/RIP FINAL_REPORTS/RIP 3.3 INFO REQUIREMENTS/F INAL_DRAFT_GUIDANCE/RIP3.3 TGD_FINAL_2007-05-02_Part1.pdf
} 


\section{Future Directions - Where do we need to go?}

The following sections briefly summarize some of the major issues considered and recommendations from the workshop designed to facilitate the development of GMP for PBPK modelling as well as identify research priorities.

\section{A. Risk Assessors needs and their role in the process}

Two possible paradigms were proposed for the involvement of the risk assessor throughout the modelling process: 1) a continuous process - wherein one would try to include any issues that the risk assessor might have while the model is being developed and 2) an iterative process - that at appropriate times, would stop and evaluate model fitness for regulatory use. The former process would occur when there is an interdisciplinary team involved in the model development and characterization (Barton et al., 2007), while the latter is typical for models that have already been published.

Risk assessors have important roles to play in mode of action and dosimetry based risk assessments utilizing PBPK models. These include helping to define the goals for using the model in the risk assessment (Clewell, H. J., 3rd et al. 2002; US EPA 2006) (Other REFs???) and participating in a transparent process that brings together appropriate interdisciplinary expertise to evaluate the model and its proposed risk assessment applications (Chiu, W. A. et al. 2007; Clark, L. H. et al. 2004). Risk assessors play a pivotal role organizing the dose-response (e.g., critical studies and endpoints) and mode of action information that form the context for applying a dosimetry model. Determining whether a PBPK model is reported to be parameterized for the chemical(s), including metabolites, species and life stages, exposure routes and matrices in the toxicity studies to be used in dose-response analysis or the human exposures relevant for the risk assessment can be accomplished by non-modellers. Identifying the dose metrics relevant to the modes of action under consideration often requires communications among risk assessors, toxicologists, and modellers as does evaluation of the biology captured by the model. Evaluation of the mathematical and computer implementation as well as 
characterization of its consistency with available data and the model's strengths and weaknesses for the proposed risk assessment applications will generally require involving people with appropriate mathematical, statistical, and computational expertise. However, to ensure a transparent process, communications describing the review process and its conclusions need to be understandable to all parties.

\section{B. Model Development Practices}

Model standardization can facilitate intra- and inter-disciplinary communications, but faces the challenges of adapting to a variety of software used to create a wide range of model structures necessary to describe different kinetic behaviors and address varying model purposes. The establishment of a standard lexicon, nomenclature and glossary would facilitate efficient communication of models and avoid confusion in semantics that can hinder understanding. There are significant benefits to the use of generic model structures; this would address the establishment of standard abbreviations or parameter nomenclature and glossary. In addition, the need to justify selected aspects of the model could be eliminated, as is currently done by citing existing literature. To be truly generic, such a model would have to encompass a wide range of physiological compartments and all useful dose metrics. A standard methodology for model building rather than a fixed model form might be an alternative (Cobelli, C. et al. 1984). The use of a hybrid of these approaches, where a simple standard model was used as a starting point and refinements that took place during the modelling workflow would be conducted with a standardized model building methodology was seen as a viable compromise. In discussing the problems caused by model code that is specific to a particular solver package, it was agreed that the use of a standard representation similar to SBML or cellML ${ }^{9}$ would improve communication between modellers and risk assessors. This type of representation gives a structured description of the conceptual model free of mathematical equations and confusing syntax. The provision of an intuitive graphical

\footnotetext{
${ }^{9}$ http://sbml.org ; www.cellml.org
} 
interface such as MEGen ${ }^{10}$ could make such standard formats more accessible to nonmodellers by allowing rapid generation of this 'PBPKML' representation.

\section{Model Characterization}

Models that have been developed sufficiently to obtain reasonable correspondence with available data can be characterized in a variety of ways to demonstrate that they are mathematically and computationally free of errors and to characterize the behavior of the model in the region of parameter space that is biologically plausible and reasonably approximates the available data (Barton, H. A. et al. 2007; Oreskes, N. 1998) (OTHER REFS???). Demonstration that a model is mathematically and computationally correctly implemented can involve checks implemented in the model (e.g., mass balance checks), rigorous manual checking of the equations and computer code, and independent recoding of the model in another software. The ease of implementing these options varies with the particular software used. A PBPK model code generator tool such as MEGen ${ }^{10}$ could play a role in both by permitting rapid recoding of models.

\section{Roles and methods of sensitivity analysis}

Sensitivity analysis is a tool for model characterization that can address a number of issues frequently raised concerning PBPK models. This was the topic of a white paper prepared for the meeting entitled "Global sensitivity analysis and its role in model development" by Dr. Martin Spendiff and Dr. George Loizou of the UK Health and Safety Laboratory, as well as being discussed in the breakout group presentation, "Fit for purpose: a proposed approach to PBPK model evaluation", given by Dr. Spendiff.

Sensitivity analyses can be used through the processes of model development, characterization, and evaluation to address issues including the following:

1. Characterize which parameters are well determined by available data.

\footnotetext{
${ }^{10}$ http://xnet.hsl.gov.uk/megen/default.aspx
} 
2. Iterating with experiments, evaluate the sensitivity of parameters to new data that will be collected. Additional, formal, experimental design methods can also be used (Cho, K.-H. et al. 2003; Gueorguieva, I. et al. 2006; Nestorov, I. A. et al. 1998).

3. For dose-response analysis predictions, evaluate the sensitivity of dose metrics predicted under the conditions relevant to the toxicity studies (or epidemiological studies) to the parameters in the model.

4. For risk assessment, evaluate the predicted dose metrics in humans under relevant environmental exposure conditions to characterize their sensitivity with respect to the model parameters.

The many sensitivity analysis methods that exist can be grouped into two categories: local methods that consider sensitivities close to a specific set of input parameter values, and global methods, which calculate the contribution of a parameter over the set of all possible input parameters. Currently, gaining insight into a model often involves the adjustment of individual model parameters and observation of the predicted changes in model output, either at a single time or throughout a time course. This useful practice can be supplemented by examining the time-dependent global sensitivities of the chosen dose-metric for dominant parameters. When trying to establish the contribution of a parameter to model predictions, local sensitivity analysis techniques are fairly rapid and simple to implement but can give somewhat misleading results if there are substantial interactions among multiple parameters. Dr. Spendiff presented an example of global sensitivity analysis using Extended Fourier Amplitude Sensitivity Test (FAST). This is a variance-based global sensitivity method that is independent of any assumptions about the model structure and is effective for monotonic (exclusively increasing or decreasing predictions) and non-monotonic models (Campolongo, F., Saltelli, A. 1997). FAST is preferable over other global methods due to its computational efficiency and capability to consider parameter interactions as well as main effects. Because PBPK models will become more complex over time, for a sensitivity analysis technique to become 'standard' for such models, it must be robust and 'future-proof'. The FAST technique satisfies these criteria. Dr. Spendiff illustrated the process with an example of a PBPK model with 57 parameters and a specified dose metric, which predicted greater than $90 \%$ 
variability across a population that was almost entirely determined by a small subset $(<10)$ of the parameters. This does not mean that the PBPK model was over-specified as it is essential that the factors responsible for heterogeneity in output across all dose metrics, species and exposure scenarios are preserved. However, once the dose metric has been established, the techniques described can greatly reduce the effort required to perform a population simulation and provide estimates of human variability.

\section{Model Documentation}

Suggestions for documenting models in publications have been presented previously (Andersen, M. E. et al. 1995). As noted there, model documentation must address a diverse readership. Recommendations from this workshop were to develop a standard, brief model description summary for the broad risk assessment audience and more detailed documentation for specialists. The summary would contain at least seven elements including:

1. Introduction with problem formulation (applicability of model),

2. Model textual description (species, routes, etc), schematic diagram, and overview of the information and data supporting the model structure

3. Metabolic pathways for the chemical and overview of supporting information and data

4. Relationship to mode of action including dose metric predictions and supporting information

5. Distributional predictions and their implications (e.g., Monte Carlo simulation of human variability)

6. Overview of uncertainty and sensitivity analyses.

7. Source of complete information (e.g., citation)

Recommendations for more complete model documentation need further development, but include the possibility that hyperlinked documents can facilitate easy access to supporting materials, including calculations done to convert published scientific information into the form utilized in the model. This extended model documentation would be utilized by subject experts in the model evaluation process and would ideally be 
publicly accessible via the internet. The documentation would strive for transparency through the use of diagrams of model structure and metabolic pathways and tables of model state variables and parameters along with the mathematical equations and model code.

\section{E. Model Evaluation}

'Best practices' allow efficient evaluation of models through standardization, documentation, and transparency. The framework for model evaluation described by Clark et al., (Clark, L. H. et al. 2004) provides a useful outline for activities in a model evaluation process that has also been extended by Chiu et al., (Chiu, W. A. et al. 2007). It would be valuable to further specify criteria that would assist reviewers in determining the strengths and limitation of a specific model. More complete specifications are needed for the processes to be used to implement model evaluation. Model evaluation needs to be a transparent, independent review process. While involvement of risk assessors and modellers throughout the steps leading from model development to application in risk assessment is valuable, it can impact the perception of the model evaluation as an independent process. An independent review is essential to identify and correct mistakes and to make judgments on the adequacy of the model and its supporting scientific database for purposes of implementing a model in risk assessment. Such reviews present a challenge for regulatory Agencies with limited PBPK modelling expertise, so it may be necessary to access additional expertise. It would also be valuable to be able to share model evaluations among countries, by agreeing upon a common framework and process even if the final decisions concerning model use might be different, for example due to risk assessment needs.

A major challenge of model evaluation is to provide perspective on the scientific uncertainties (i.e., inexact or incomplete information) identified with the model and its supporting scientific database. The models allow characterization of uncertainty in a way that default analyses cannot. For example, a default value of 10 for interspecies extrapolation is commonly applied, but the uncertainty for any specific chemical with 
regard to the toxicity it causes in animals ranges from a value close to zero (the effect only occurs in the animals) to a much larger value (the effect only occurs in humans). While the factor of 10 represents a judgment concerning the general tendency across many chemicals, it cannot describe the uncertainties for a specific chemical as one can start to estimate using biologically based modelling. However, this creates a challenge for considering whether the model adequately captures the science and, thus, should be implemented in the risk assessment.

\section{Improving Science Supporting Models}

Efforts to use PBPK models more broadly have also resulted in a range of scientific issues that require additional research. These include improving methods for using in vitro data in order to limit controlled animal and human studies, for model development by extrapolating across chemical to those with limited databases, and for better characterizing uncertainty and variability in PBPK models.

\section{In vitro to in vivo extrapolations}

During the presentation by Professor György Csanády of the GSF- Institute of Toxicology in Germany entitled "A physiological toxicokinetic model for inhaled propylene oxide in rat and human with special emphasis on the nose" he discussed the significant capabilities of this model given the complex nature of the toxicity and datasets it was addressing. He noted the apparent inability of the model to predict in vivo rat data using metabolic parameters estimated from in vitro studies (Csanady, G. A. and J. G. Filser 2007; Faller, T. H. et al. 2001; Lee, M. S. et al. 2005; Osterman-Golkar, S. et al. 2003). Introduction of extrahepatic metabolism or reduction of the Michaelis-Menten $K_{M}$ constant was required, while a reduction in pulmonary ventilation rate was need at propylene oxide concentrations above 100 ppm likely reflecting respiratory irritant effects. The discussion stimulated by Professor Csanády's presentation led to agreement that the issue of in vitro to in vivo extrapolation, particularly, with regards to metabolism requires further detailed study (Blaauboer, B. J. et al. 1999; Blaauboer, B. J. et al. 1996; DeJongh, J. et al. 1999). Ideally, in vitro data should be readily usable in PBPK models 
because it can limit the need for in vivo studies in animals or humans. The importance of protein and non-specific binding and partitioning of volatile substrates, in particular, between the liquid and headspace phases are fundamental to improving the utility of in vitro systems and the use of such data in PBPK models. The EU $6{ }^{\text {th }}$ Framework Project "AcuteTox" is addressing many of these issues ${ }^{11}$. Therefore, in vitro metabolism data currently must be ranked lower than in vivo until more detailed models of in vitro systems demonstrate that they are reliable surrogates. In keeping with the theme of the workshop it was agreed that transparency and clear communication with regard to how in vitro data were generated and used in a model is vital.

\section{Cross chemical extrapolation}

Risk assessors are increasingly having to address prioritization and assessment for the large numbers of chemicals in commerce, notably the REACH ${ }^{12}$ legislation in Europe or the Categorization \& Screening of the Domestic Substances List under the Canadian Environmental Protection Act of $1999^{13}$. Methods to develop initial PBPK models for chemicals using cross-chemical prediction methods would be valuable. Efforts to date have primarily been directed at predicting tissue:blood or tissue:air partition coefficients (Beliveau, M. et al. 2005), though in vitro to in vivo extrapolation for metabolism and other aspects of pharmacokinetics is also receiving attention as noted in the previous section.

\section{Uncertainty and variability in PBPK models}

Much of the focus in the development of PBPK models has been to identify and capture the average behaviour of the key biological processes controlling a chemical's pharmacokinetics. These models have successfully assisted in evaluating biological hypotheses for mode of action (e.g., methylene chloride carcinogenesis described previously) as well as identifying previously unrecognized pharmacokinetic behaviours. The increasing application of PBPK models in risk assessment has led to a range of efforts to better characterize the relationship between the model and supporting data and

\footnotetext{
11 (http://www.acutetox.org/)

12 (http://ec.europa.eu/environment/chemicals/reach/reach_intro.htm )

13 (http://www.ec.gc.ca/substances)
} 
quantify uncertainty and variability as was described by Dr. R. Woodrow Setzer in his talk reporting on the International Workshop on Uncertainty and Variability in PBPK Models (Research Triangle Park, NC, 31 October - 2 November, 2006). Improved computing power was essential to more widespread use of distributional analyses to characterize human variability with Monte Carlo simulation techniques and methods of parameter estimation ranging from optimization of selected chemical specific parameters (e.g., metabolic rates) to global parameter estimation using Bayesian statistical characterization of uncertainty and variability. Priorities for research and implementation of uncertainty and variability concepts in risk assessments using PBPK models have been previously described (Barton et al., 2007).

\section{G. Good modelling practices for PBPK models: Developing a description, case studies, and training materials}

The International Programme on Chemical Safety (IPCS) steering group of the World Health Organization (WHO) identified PBPK modelling as an important component of chemical risk assessment that merits international harmonization ${ }^{14}$. The ability to review a PBPK model according to accepted criteria would greatly facilitate widespread acceptance, in particular, amongst regulators. While agreement amongst PBPK practitioners is paramount for the development of GMP, the guidelines must also be acceptable to regulators and risk assessors. Development of guidelines for GMP is best achieved through a cross-disciplinary exchange of experience and ideas among laboratory scientists, PBPK modellers, regulators and risk assessors. This workshop will provide input to the ongoing IPCS PBPK working group, which is striving to develop a description of GMP.

The adequacy of the GMP description can be evaluated using case studies. The case studies would then form the basis for training materials on GMPs. Some recommendations for case studies included:

\footnotetext{
${ }^{14}$ http://www.who.int/ipcs/methods/harmonization/areas/pbpk/en/index.html
} 
- Comparing a case with a dose metric for which data was directly available versus one where it was not.

- Examples where PBPK models were accepted and used by regulatory Agencies and ones where they were rejected to insure appropriate documentation.

- Comparisons of data rich chemicals with data limited chemicals including not just pharmacokinetic or metabolic data, but also mode of action data such as toxicogenomic or metabolomic data.

- Illustrations of different risk assessment applications

Potential chemicals to use as case studies would include those previously noted by participants as PBPK models considered for or applied in risk assessments in Europe, Canada, and the United States. Other chemicals could include isopropanol (with acetone metabolite submodel) for noncancer endpoints, styrene as an example of an inaccessible dose metric, acrylamide as an example of great current regulatory interest with multiple proposed modes of action and target sites, butadiene due to the substantial animal modelling and uncertainty in human metabolism resulting in assessment based upon epidemiology.

Finally, development of training materials and hiring of personnel with needed expertise will be essential to facilitate implementation of mode of action and dosimetry-based risk assessment by regulatory Agencies. A strategy over a longer term would be to include a more quantitative, computationally based study of toxicology in university courses. The adaptation of a PBPK model generator tool such as MEGen as a teaching tool would be very useful in demonstrating to students how biological knowledge can be applied to solve real-world problems. Training materials are needed so that risk assessors and managers with diverse expertise can successfully interact with modellers to implement PBPK models in risk assessment. Training will also be important for modellers to learn about newer methodologies for characterizing uncertainty and variability in PBPK models or implementing local and global sensitivity analyses at appropriate stages of model maturation. 


\section{REFERENCES}

Allen, B. C., Covington, T. R., and Clewell, H. J. (1996). Investigation of the impact of pharmacokinetic variability and uncertainty on risks predicted with a pharmacokinetic model for chloroform. Toxicol. 111, 289-303.

Andersen, M. E., Clewell III, H. J., and Frederick, C. B. (1995). Applying simulation modeling to problems in toxicology and risk assessment - a short perspective. Toxicol. Appl. Pharmacol. 133, 181-187.

Babich, M. A. (1998). Risk assessment of low-level chemical exposures from consumer products under the U.S. Consumer Product Safety Commission chronic hazard guidelines. Environ Health Perspect 106 Suppl 1, 387-90.

Barratt, M. D., Castell, J. V., Chamberlain, M., Combes, R. D., Dearden, J. C., Fentem, J. H., Gerner, I., Giuliani, A., Gray, T. J. B., Livingstone, D. J., Provan, W. M. L., Rutten, F., Verhaar, H. J. M., and Zbinden, P. (1995). The Integrated Use of Alternative Approaches for Predicting Toxic Hazard - the Report and Recommendations of Ecvam Workshop-8. ATLA-Altern. Lab. Anim. 23, 410-429.

Barton, H. A., Chiu, W. A., Setzer, R. W., Andersen, M. E., Bailer, A. J., Bois, F. Y., Dewoskin, R. S., Hays, S., Johanson, G., Jones, N., Loizou, G., Macphail, R. C., Portier, C. J., Spendiff, M., and Tan, Y. M. (2007). Characterizing Uncertainty and Variability in Physiologically-based Pharmacokinetic (PBPK) Models: State of the Science and Needs for Research and Implementation. Toxicol Sci, aheadofprint.

Barton, H. A., Flemming, C. D., and Lipscomb, J. C. (1996). Evaluating human variability in chemical risk assessment: hazard identification and dose-response assessment for noncancer oral toxicity of trichloroethylene. Toxicol. 111, 271-87.

Beliveau, M., Lipscomb, J., Tardif, R., and Krishnan, K. (2005). Quantitative StructureProperty Relationships for Interspecies Extrapolation of the Inhalation Pharmacokinetics of Organic Chemicals. Chem Res Toxicol 18, 475-485.

Blaauboer, B. J., Barratt, M. D., and Houston, B. J. (1999). The Integrated Use of Alternative Methods in Toxicological Risk Evaluation: ECVAM Integrated Testing Strategies Task Force Report 1. ATLA-Altern. Lab. Anim. 27, 229-237.

Blaauboer, B. J., Bayliss, M. K., Castell, J. V., Evelo, C. T. A., Frazier, J. M., Groen, K., Gülden, M., Guillouzo, A., Hissink, A. M., Houston, B. J., Johanson, G., de Jongh, J., Kedderis, G. L., Reinhardt, C. A., van de Sandt, J. J. M., and Semino, G. (1996). The use of Biokinetics and In Vitro Methods in Toxicological Risk Evaluation. ATLA 24, 473-497.

Boobis, A. R., Cohen, S. M., Dellarco, V., McGregor, D., Meek, M. E., Vickers, C., Willcocks, D., and Farland, W. (2006). IPCS Framework for Analyzing the Relevance of a Cancer Mode of Action for Humans. Crit Rev Toxicol 36, 781-92. 
Boobis, A. R., Doe, J. E., Heinrich-Hirsch, B., Meek, M. E., Munn, S., Ruchirawat, M., Schlatter, J., Seed, J., and Vickers, C. (2007). IPCS framework for analysing the relevance of a non-cancer mode of action for humans. Crit Rev Toxicol in press.

Campolongo, F., Saltelli, A. (1997). Sensitivity analysis of an environmental model; a worked application of different analysis methods. Reliability Engineering and System Safety, 49-69.

Chiu, W. A., Barton, H. A., Dewoskin, R. S., Schlosser, P., Thompson, C. M., Sonawane, B., Lipscomb, J. C., and Krishnan, K. (2007). Evaluation of physiologically based pharmacokinetic models for use in risk assessment. J Appl Toxicol 27, 218-237.

Chiu, W. A., Caldwell, J. C., Keshava, N., and Scott, C. S. (2006). Key scientific issues in the health risk assessment of trichloroethylene. Environ Health Perspect 114, 1445-9.

Cho, K.-H., Shin, S.-Y., Kolch, W., and Wolkenhauer, O. (2003). Experimental Design in Systems Biology, Based on Parameter Sensitivity Analysis Using a Monte Carlo Method: A Case Study for the TNF $\{$ alpha $\}$-Mediated NF-\{kappa $\}$ B Signal Transduction Pathway. SIMULATION 79, 726-739.

Clark, L. H., Setzer, R. W., and Barton, H. A. (2004). Framework for evaluation of physiologically-based pharmacokinetic models for use in safety or risk assessment. Risk Anal 24, 1697-717.

Clewell, H. J., 3rd, Andersen, M. E., and Barton, H. A. (2002). A consistent approach for the application of pharmacokinetic modeling in cancer and noncancer risk assessment. Environ. Health Perspect. 110, 85-93.

Clewell, H. J., 3rd, Andersen, M. E., Wills, R. J., and Latriano, L. (1997). A physiologically based pharmacokinetic model for retinoic acid and its metabolites. $\mathrm{J} \mathrm{Am}$ Acad Dermatol 36, S77-85.

Clewell, H. J., Gearhart, J. M., Gentry, P. R., Covington, T. R., VanLandingham, C. B., Crump, K. S., and Shipp, A. M. (1999). Evaluation of the uncertainty in an oral reference dose for methylmercury due to interindividual variability in pharmacokinetics. Risk Anal 19, 547-58.

Clewell, H. J., Teeguarden, J., McDonald, T., Sarangapani, R., Lawrence, G., Covington, T., Gentry, R., and Shipp, A. (2002). Review and evaluation of the potential impact of age- and gender-specific pharmacokinetic differences on tissue dosimetry. Crit Rev Toxicol 32, 329-89.

Clewell III, H. J., and Andersen, M. E. (1987). Dose, species and route extrapolation using physiologically-based pharmacokinetic modeling. Drinking Water and Health $\mathbf{8}$, 159-184.

Cobelli, C., Carson, E. R., Finkelstein, L., and Leaning, M. S. (1984). Validation of simple and complex models in physiology and medicine. Am J Physiol 246, R259-66. 
Cox, L. A., Jr. (1996). Reassessing benzene risks using internal doses and Monte-Carlo uncertainty analysis. Environ Health Perspect 104 Suppl 6, 1413-29.

Csanady, G. A., and Filser, J. G. (2007). A physiological toxicokinetic model for inhaled propylene oxide in rat and human with special emphasis on the nose. Toxicol Sci 95, 3762.

DeJongh, J., Forsby, A., Houston, J. B., Beckman, M., Combes, R., and Blaauboer, B. J. (1999). An integrated approach to the prediction of systemic toxicity using computerbased biokinetic models and biological in vitro test methods: overview of a prevalidation study based on the ECITTS Project. Toxicol In Vitro 13, 549-554.

Delic, J. I., Lilly, P. D., MacDonald, A. J., and Loizou, G. D. (2000). The utility of PBPK in the safety assessment of chloroform and carbon tetrachloride. Regul Toxicol Pharmacol 32, 144-55.

Dewoskin, R. S. (2007). PBPK models in risk assessment-A focus on chloroprene. Chem Biol Interact, aheadofprint.

Dewoskin, R. S., Lipscomb, J. C., Thompson, C., Chiu, W. A., Schlosser, P., Smallwood, C., Swartout, J., Teuschler, L. K., and Marcus, A. (2006).

Pharmacokinetic/Physiologically Based Pharmacokinetic Models in Integrated Risk Information System Assessments. In Toxicokinetics and Risk Assessment (J. C.

Lipscomb and E. V. Ohanian, eds.), pp. 301-348. Informa Healthcare USA, Inc, New York.

Faller, T. H., Csanady, G. A., Kreuzer, P. E., Baur, C. M., and Filser, J. G. (2001). Kinetics of propylene oxide metabolism in microsomes and cytosol of different organs from mouse, rat, and humans. Toxicol Appl Pharmacol 172, 62-74.

Franks, S. J. (2005). A mathematical model for the absorption and metabolism of formaldehyde vapour by humans. Toxicol Appl Pharmacol 206, 309-20.

Franks, S. J., Spendiff, M. K., Cocker, J., and Loizou, G. D. (2006). Physiologically based pharmacokinetic modelling of human exposure to 2-butoxyethanol. Toxicol Lett 162, 164-73.

Georgopoulos, P. G., Roy, A., and Gallo, M. A. (1994). Reconstruction of short-term multi-route exposure to volatile organic compounds using physiologically based pharmacokinetic models. J. Expos. Anal. Environ. Epidem. 4, 309-328.

Gueorguieva, I., Aarons, L., Ogungbenro, K., Jorga, K. M., Rodgers, T., and Rowland, M. (2006). Optimal design for multivariate response pharmacokinetic models. $J$ Pharmacokinet Pharmacodyn 33, 97-124.

Gundert-Remy, U., and Sonich-Mullin, C. (2002). The use of toxicokinetic and toxicodynamic data in risk assessment: an international perspective. Sci Total Environ 288, 3-11. 
Hays, S. M., Becker, R. A., Leung, H. W., Aylward, L. L., and Pyatt, D. W. (2007). Biomonitoring equivalents: a screening approach for interpreting biomonitoring results from a public health risk perspective. Regul Toxicol Pharmacol 47, 96-109.

IPCS (2005). Chemical-Specific Adjustment Factors (CSAFs) For Interspecies Differences And Human Variability: Guidance Document For Use Of Data In Dose/Concentration-Response Assessment, p. 96 http://whqlibdoc.who.int/publications/2005/9241546786_eng.pdf. WHO, Geneva.

Lee, M. S., Faller, T. H., Kreuzer, P. E., Kessler, W., Csanady, G. A., Putz, C., RiosBlanco, M. N., Pottenger, L. H., Segerback, D., Osterman-Golkar, S., Swenberg, J. A., and Filser, J. G. (2005). Propylene oxide in blood and soluble nonprotein thiols in nasal mucosa and other tissues of male Fischer $344 / \mathrm{N}$ rats exposed to propylene oxide vapors-relevance of glutathione depletion for propylene oxide-induced rat nasal tumors. Toxicol Sci 83, 177-89.

Meek, B., Renwick, A., and Sonich-Mullin, C. (2003). Practical application of kinetic data in risk assessment--an IPCS initiative. Toxicol Lett 138, 151-60.

Meek, M. E., Bucher, J. R., Cohen, S. M., Dellarco, V., Hill, R. N., Lehman-McKeeman, L. D., Longfellow, D. G., Pastoor, T., Seed, J., and Patton, D. E. (2003). A framework for human relevance analysis of information on carcinogenic modes of action. Crit Rev Toxicol 33, 591-653.

Meek, M. E., and Renwick, A., eds. (2006). Guidance for the development of chemical specific adjustment factors - integration with mode of action frameworks. Informa Healthcare, New York.

Meek, M. E., Renwick, A., Ohanian, E., Dourson, M., Lake, B., Naumann, B. D., and Vu, V. (2001). Guidelines for application of chemical specific adjustment factors (CSAF) in dose/concentration response assessment. Comments in Toxicology 7, 575-590.

Meek, M. E., Renwick, A., Ohanian, E., Dourson, M., Lake, B., Naumann, B. D., and Vu, V. (2002). Guidelines for application of chemical-specific adjustment factors in dose/concentration-response assessment. Toxicol. 181-182, 115-20.

Nestorov, I. A., Aarons, L. J., Arundel, P. A., and Rowland, M. (1998). Lumping of whole-body physiologically based pharmacokinetic models. J Pharmacokinet Biopharm 26, 21-46.

Oreskes, N. (1998). Evaluation (not validation) of quantitative models. Environ Health Perspect 106 Suppl 6, 1453-60.

OSHA (1997). Occupational Exposure to methylene chloride; Final Rule 20 CFR Parts 1910, 1915 and 1926 70FR1493-1619. http://www.osha.gov/SLTC/methylenechloride Accessed August 1, 2007. 
Osterman-Golkar, S., Czene, K., Lee, M. S., Faller, T. H., Csanady, G. A., Kessler, W., Perez, H. L., Filser, J. G., and Segerback, D. (2003). Dosimetry by means of DNA and hemoglobin adducts in propylene oxide-exposed rats. Toxicol Appl Pharmacol 191, 24554.

Portier, C. J., and Lyles, C. M. (1996). Practicing safe modeling: GLP for biologically based mechanistic models. Environ Health Perspect 104, 806.

Rescigno, A., and Beck, J. S. (1987). The use and abuse of models. J Pharmacokinet Biopharm 15, 327-44.

Rowland, M., Balant, L., and Peck, C. (2004). Physiologically Based Pharmacokinetics in Drug Development and Regulatory Science: A Workshop Report (Georgetown University, Washington, DC, May 29-30, 2002). AAPS PharmSci 6, 1-12.

Seed, J., Carney, E. W., Corley, R. A., Crofton, K. M., DeSesso, J. M., Foster, P. M., Kavlock, R., Kimmel, G., Klaunig, J., Meek, M. E., Preston, R. J., Slikker, W., Jr., Tabacova, S., Williams, G. M., Wiltse, J., Zoeller, R. T., Fenner-Crisp, P., and Patton, D. E. (2005). Overview: Using mode of action and life stage information to evaluate the human relevance of animal toxicity data. Crit Rev Toxicol 35, 664-72.

Sonich-Mullin, C., Fielder, R., Wiltse, J., Baetcke, K., Dempsey, J., Fenner-Crisp, P., Grant, D., Hartley, M., Knaap, A., Kroese, D., Mangelsdorf, I., Meek, E., Rice, J. M., and Younes, M. (2001). IPCS conceptual framework for evaluating a mode of action for chemical carcinogenesis. Regul Toxicol Pharmacol 34, 146-52.

US EPA (1987). Update to the Health Assessment Document and Addendum for Dichloromethane (Methylene Chloride): Pharmacokinetics, Mechanism of Action, and Epidemiology. External Review Draft. EPA/600/8-87/030A.

US EPA (1989). Risk Assessment Guidance for Superfund. Volume 1. Human Health Evaluation Manual (Part A). EPA/540/1-89/002.

US EPA (2005). Guidelines for Carcinogen Risk Assessment (Final). EPA/630/P03/001F.

US EPA (2006). Approaches for the Application of Physiologically Based Pharmacokinetic (PBPK) Models and Supporting Data in Risk Assessment (Final Report). EPA/600/R-05/043A. 